\title{
Candida Skull Base Osteomyelitis: a Case Report and Literature Review
}

\author{
Madhusudhan Krishnamoorthy, ${ }^{1, *}$, Nik Adilah Nik Othman¹, Nor Eyzawiah binti Hassan ${ }^{2,3}$, \\ Shahrul bin Hitam ${ }^{3}$
}

\begin{abstract}
Skull base osteomyelitis (SBO) also commonly known as malignant otitis externa was first described by Meltzer and Kelemen in 1959. Prior to the advent of the antibiotic era, this disease carried a poor prognosis with significant morbidity. It often proved fatal with mortality rates as high as $50 \%$. Commonly seen in the immunocompromised patients, diabetes mellitus is an important associated comorbidity in the pathophysiologic development of this disease. Treatment is instituted by medical therapy with surgery having a limited role. Surgical intervention has a limited role, for example, in fungal SBO. Such cases may require local debridement and intraoperative tissue biopsies for histopathologic confirmation. This is to demonstrate fungal invasion into the skull base, as well as to exclude other sinister differential diagnoses like squamous cell carcinoma of temporal bone. In this case report, we present a rare case of candida SBO and the literature review.
\end{abstract}

\section{KEYWORDS}

skull base; osteomyelitis; fungal; candida; otitis externa

\author{
AUTHOR AFFILIATIONS \\ ${ }^{1}$ Department of Otorhinolaryngology and Head \& Neck Surgery, Hospital Universiti Sains Malaysia, School of Medical Sciences, Kubang \\ Kerian, Kelantan, Malaysia \\ ${ }^{2}$ Faculty of Medicine and Health Sciences, Universiti Sains Islam Malaysia (USIM), Pandan Indah, Selangor, Malaysia \\ ${ }^{3}$ Department of Otorhinolaryngology and Head \& Neck Surgery, Hospital Ampang, Selangor, Malaysia \\ * Corresponding author: Department of Otorhinolaryngology and Head \& Neck Surgery, Hospital Universiti Sains Malaysia, School of \\ Medical Sciences, Kubang Kerian, Kelantan, Malaysia; e-mail: kmadhu_87@yahoo.com
}

Received: 15 November 2019

Accepted: 22 May 2020

Published online: 10 August 2020

Acta Medica (Hradec Králové) 2020; 63(2): 82-85

https://doi.org/10.14712/18059694.2020.22

(c) 2020 The Authors. This is an open-access article distributed under the terms of the Creative Commons Attribution License (http://creativecommons.org/licenses/by/4.0), which permits unrestricted use, distribution, and reproduction in any medium, provided the original author and source are credited. 


\section{INTRODUCTION}

Skull base osteomyelitis (SBO) is usually caused by Pseudomonas aeruginosa (1). Various other microorganisms such as fungi may attribute to the development of SBO (2). A common fungal pathogen responsible is the Aspergillus species (3). Our patient, an elderly diabetic, presented with classical symptoms of nocturnal excruciating otalgia and ear discharge. On further investigation however, the etiologic agent was found to be Candida species.

\section{CASE PRESENTATION}

Informed consent was taken from the patient prior to the commencement of clinical examination, investigation and treatment.

A 60-year-old male presented to us with history of right sided earache associated with ear discharge of oneweek duration. The otalgia was described as nocturnal and excruciating in nature, disturbing his sleep. There was no history of reduced hearing, and no complaints of vertigo. Otherwise, there were no complaints over the left ear. He had long standing diabetes mellitus of approximately 20 years requiring insulin therapy for optimization of blood sugar levels. Due to the unsatisfactory diabetic control, he suffered from chronic kidney disease however he did not require renal replacement therapy. He also had a left sided below knee amputation 1-year prior as complications of his poorly managed sugar levels. Clinical examination revealed a narrowed and edematous ear canal, with presence of granulation tissue along the floor of the ear canal. The tympanic membrane was intact, however dull in appearance. He was treated as skull base osteomyelitis with topical ciprofloxacin drops (Tarivid Otic; Daiichi Sankyo, Tokyo, Japan) and systemic ciprofloxacin tablets (C-Flox; Intas Pharmaceuticals, Ahmedabad, India). There was no compromise in any of the cranial nerves functions, espe-

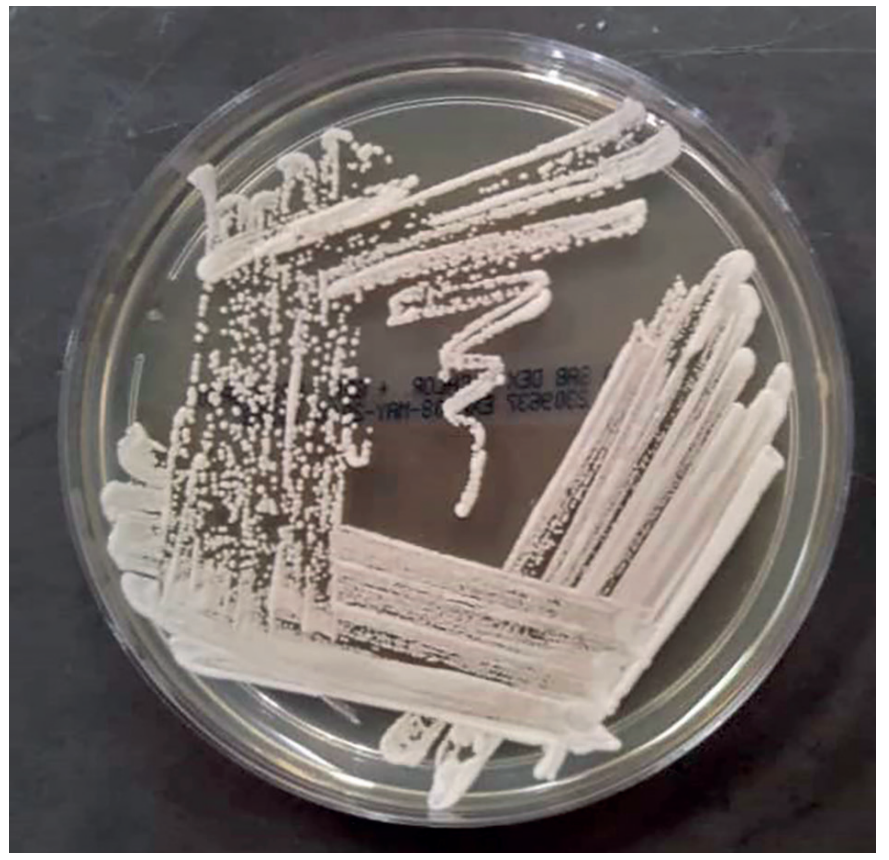

Fig. 1 Candida albicans culture. cially the facial nerve. Patient was managed on an outpatient basis as his compliance to medication was good, and he was monitored on a fortnightly basis.

Eight weeks after his first presentation, he complained of a painless right sided facial swelling. Clinical examination revealed a vague right pre-auricular fullness, approximately $4 \times 3 \mathrm{~cm}$, circular shaped, smooth surfaced, with ill-defined margins. Otoendoscopy of the right ear revealed a narrowed ear canal, with granulation tissue along the floor of the canal, just like the initial presentation. Patient was admitted for treatment with empirical intravenous ciprofloxacin (Ciproxol; Ain Medicare, Kota Bharu, Malaysia) antibiotics (dosed adjusted based on the underlying impaired kidney functions to prevent nephrotoxicity). His blood investigations revealed hemoglobin of $9.6 \mathrm{~g} / \mathrm{dL}$, total white cell count of $10.9 \mathrm{~K} / \mu \mathrm{L}$, urea $8.9 \mathrm{mmol} / \mathrm{L}$, and creatinine of $205 \mathrm{mmol} / \mathrm{L}$. His ESR (erythrocyte sedimentation rate) was elevated, $90 \mathrm{~mm} /$ hour. A swab taken from the ear for culture and sensitivity prior to commencement of antibiotics confirmed Candida albicans infection (Figure 1). He was then treated with systemic anti-fungal (Fluconazole; Pharmaniaga, Bangi, Malaysia) therapy. Biopsies taken from the granulation tissue were confirmatory for inflammatory granulation tissue and ruled out malignancy. There was no fungal hyphae or spores seen on histopathologic examination. An urgent high-resolution computed tomography (HRCT) of the temporal bone was done (Somatom Perspective syngo CT VC30 machine; Siemens, Erlangen, Germany). There was bony erosion along the greater wing of the right sphenoid with osteomyelitic changes (Figure 2). There was also erosion of the anterior wall of the right ear canal (Figure 3). The stylomastoid foramen and adjacent stylomastoid process showed inflammation (Figure 4). The right middle ear cavity and mastoid air cells were filled with soft tissue densities, however there was no destruction of the ossicles or the scutum (Figure 5). After 2 weeks of medical therapy using antimicrobials, there was marked resolution in otalgia, and he was able to sleep well

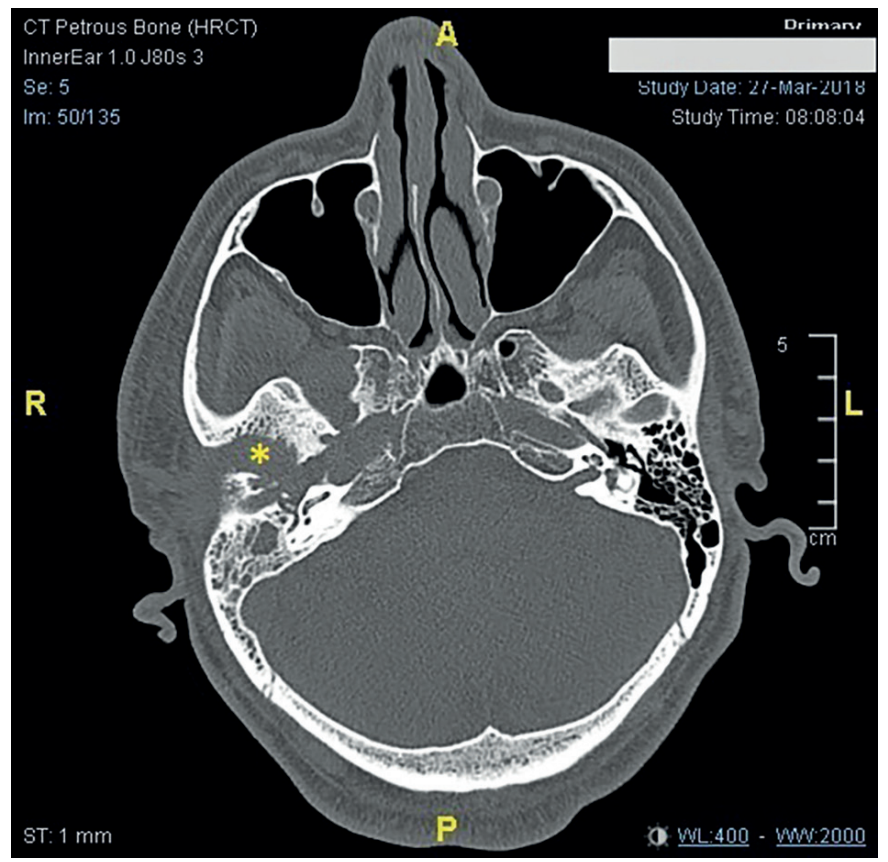

Fig. 2 Erosions along the greater wing of right sphenoid marked as* 


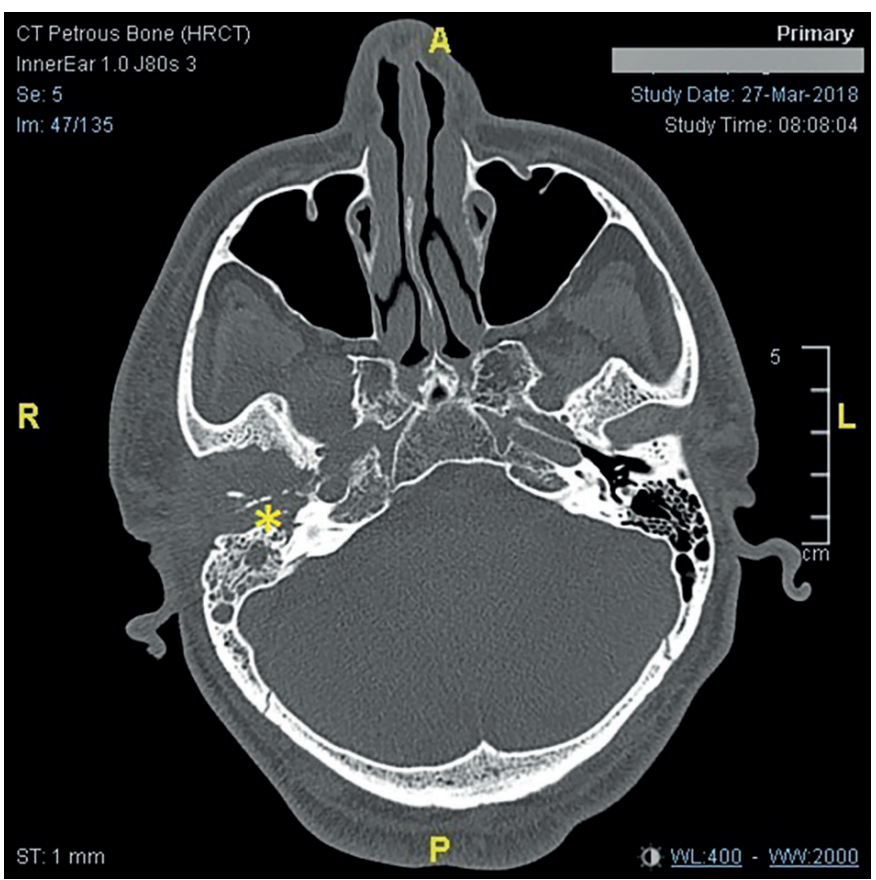

Fig. 3 Erosions along the anterior wall of right external auditory canal marked as*.

at night. Patient was satisfied with his treatment outcome and was discharged home well.

During his one-week post discharge review, the granulation tissue was noted to persist (Figure 6) despite patient's compliance to daily anti-fungal therapy (fluconazole tablets). Patient was advised for a repeat biopsy to rule out other sinister pathologies such as squamous cell carcinoma of the temporal bone, and for surgical intervention, but he refused. Subsequently, he did turn up for his following appointment.

\section{DISCUSSION}

The first documentation of progressive temporal bone osteomyelitis dates back to 1838 by Toulmouche (3). Otogenic SBO was first described by Meltzer and Kelemen in 1959 (4). The term malignant otitis externa is a misnomer as it does not define a neoplastic disease (5) and this term is used interchangeably with SBO. It was coined by Chandler in 1968 to describe the aggressive nature of destruction caused by Pseudomonas aeruginosa in the ear canal and spreading infection to the skull base (6). Later in 1985, Petrak et al. described the first case of fungal malignant otitis externa (7). Risk factors attributable to the development of SBO include, elderly age of more than 60 years, diabetes mellitus and an immunocompromised state. Diabetes mellitus is an important associated comorbidity in SBO patients, with a prevalence as high as $90-100 \%$ of all SBO patients (8). Various other conditions predisposing to SBO are Human Immunodeficiency Virus (HIV) infection, chemotherapy induced aplasia and chronic leukemia $(7,8)$. In fungal SBO patients, more than $70 \%$ have underlying diabetes mellitus (2). There are a few routes of fungal infection spread in the pathogenesis of SBO. They are i) via the external ear canal, the infection spreads through the Santorini fissures and medially to the tympanomastoid

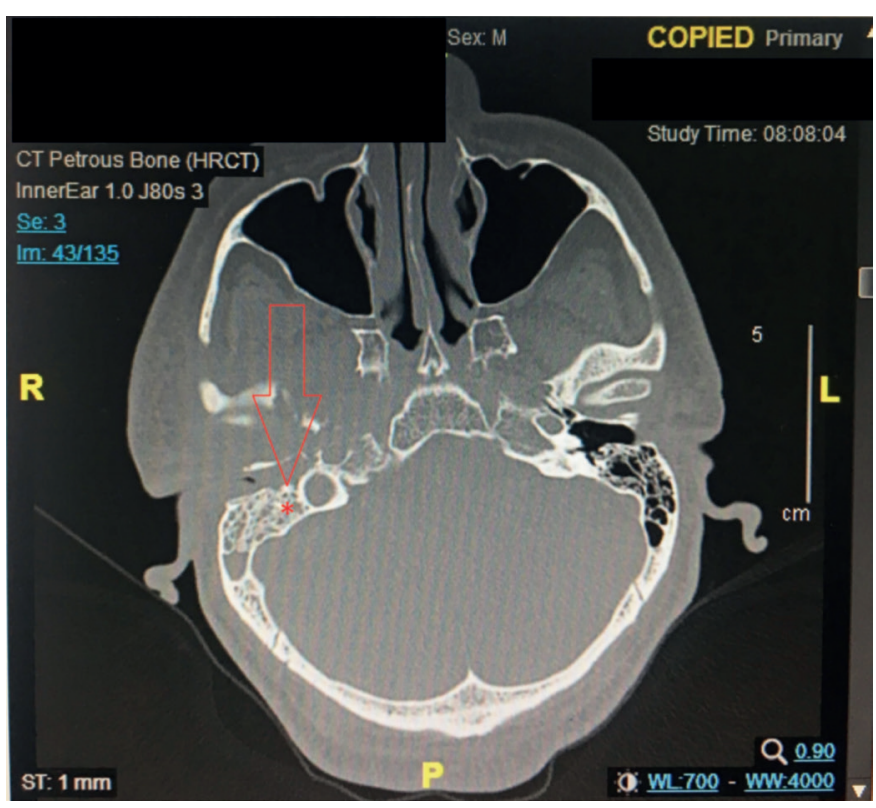

Fig. 4 Inflammation around the stylomastoid foramen and stylomastoid process.

suture $(6,8)$,ii) via the internal acoustic meatus in cases of fungal meningitis, iii) via the Eustachian tube and iv) via hematogenic spread. Very rarely, it follows a paranasal sinus fungal infection (6). The reason for developing SBO in diabetic patients is attributable to the endarteritis and microangiopathy causing small vessel obliteration (8).

Although the most common etiologic agent for fungal SBO is Aspergillus fumigatus $(6,8)$, cases of Candida species similar to our case have been reported in literature $(1,9)$. A case of proven (definite) SBO is defined as skull base infection with symptoms and signs localizing at presentation, with, radiologic evidence of bone erosion and isolation of organism from the affected bone. A probable SBO however, is when the organism is isolated from a source other than bone or tissue (for example, from an ear swab) (2). As seen in our case, fungal SBO is more rapid to present (mean time to presentation of 8 weeks, as compared to 26 weeks for bacterial SBO) (2). The typical presentation is a patient who is 60 years old or elder, with underlying diabetes complaining of unremitting earache and headache. Classically fever is absent. They may also present with a swelling in the preauricular region (4). Blood investigations reveal absent leukocytosis with markedly elevated erythrocyte sedimentation rate (ESR) $(8-10)$. In severe cases, cranial nerve palsy occurs, commonest being the facial nerve (cranial nerve VII) $(2,8,9)$ due to the proximity of the ear canal to the stylomastoid foramen. The next common group of cranial nerves to be involved are the nerves in the jugular foramen, namely the glossopharyngeal, the vagus and the spinal accessory (cranial nerves IX, X and XI respectively) (8).Clinical examination usually reveals granulation tissue in the ear canal. Our case was similar to the other reported literatures where ear swab grew Candida albicans and biopsy of granulation tissue taken from the ear canal was negative for malignancy $(4,9,10)$. This presence of granulation tissue seems to indicate a good treatment outcome in fungal SBO (7). However, it is important to remember that in 
Tab. 1 Etiology of origin for SBO.

\begin{tabular}{|c|c|c|c|c|c|c|}
\hline \multicolumn{7}{|c|}{ Skull base osteomyelitis } \\
\hline \multicolumn{3}{|c|}{ Sinorhino - otogenic origin } & \multicolumn{4}{|c|}{ Non Sinorhino - otogenic origin } \\
\hline Anterior skull base & Middle skull base & Posterior skull base & Trauma & latrogenic & Hematogenous & Others \\
\hline
\end{tabular}

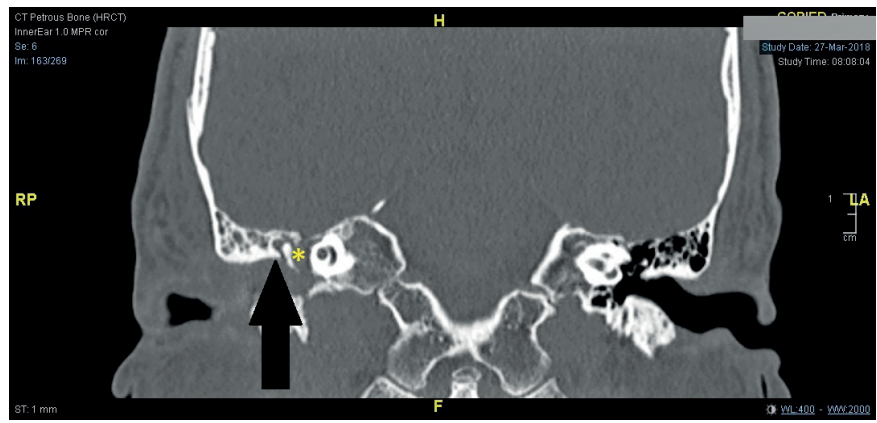

Fig. 5 Soft tissue density in the middle ear cavity and mastoid air cells, neither violating the scutum (black arrow) nor violating the ossicles $(*)$.

cases of recalcitrant disease not responding to treatment, a differential diagnosis of squamous cell carcinoma of the temporal bone must be born in mind. This is because cases of early carcinomas may be misleading and such cases may be presumed to be as SBO (5). Therefore, there is a role of tissue biopsy under general anesthesia for cases of probable SBO.

$\mathrm{SBO}$ can be classified based on etiologic origin as seen in Table 1 (3). Fungal SBO infection usually originates from the middle ear cavity or the mastoid air cells (6-8). In our case report, HRCT imaging showed evidence of middle ear destruction with soft tissue density seen in the mastoid air cell system $(9,10)$, however the ossicles and scutum were not violated. HRCT is sensitive to detect bony erosions and defects, however inadequate to monitor treatment response. As such other imaging modalities are needed such as Technetium 99m-methyl diphosphonate (Tc-99m Mdp) bone scan as well as Gallium scan. Both of these modalities are complementary to diagnose and monitor SBO (8). Treatment constitutes prolonged antibiotic therapy (6) with quinolones due to good bioavailability and effective bone penetration. Aggressive control of blood sugar levels is also crucial. In cases of fungal SBO systemic as well as topical antifungals are required in medical treatment $(9,10)$. There is a role of surgical therapy in fungal SBO (2), however its role is limited to biopsy, debridement and drainage of abscesses $(3,8)$. Hyperbaric oxygen therapy has been documented in literature as a useful adjunct, however due to the restricted availability and minimal evidence based results, it is not a common practice $(3,9)$. Long term survival is based on severity of disease, rather than a particular therapeutic regime (7). Prognosis is largely dependent on the underlying immune status of the patient (6).

\section{CONCLUSION}

$\mathrm{SBO}$ is an uncommon disease, usually resulting as a complication from an uncontrolled otogenic, odontogenic or sinus infection. Almost half (48\%) of SBO patients may develop

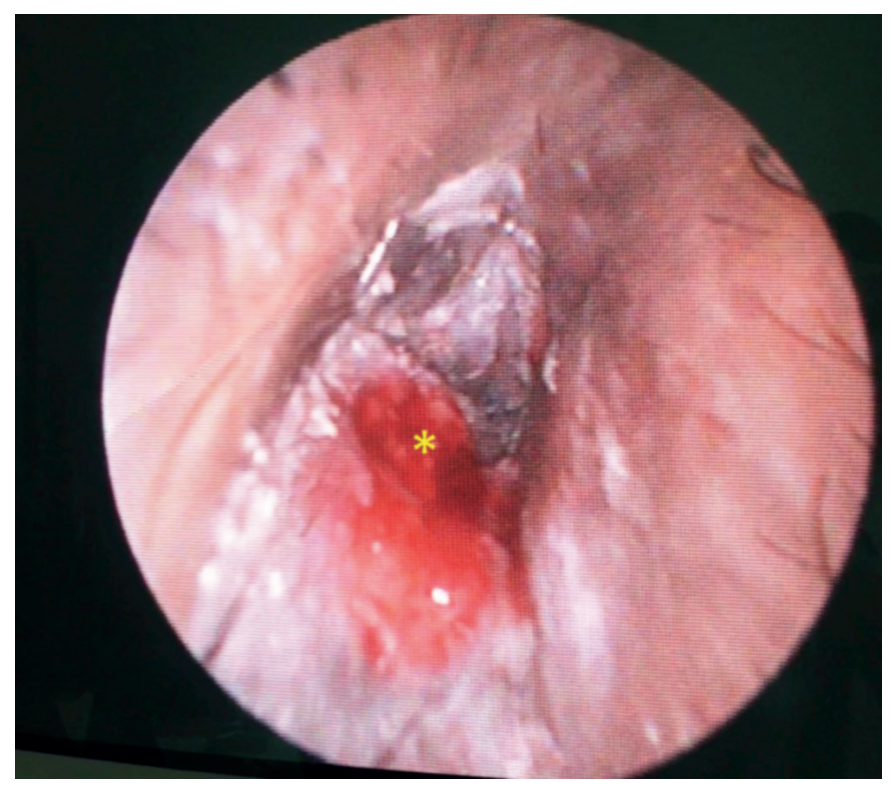

Fig. 6 Persistent granulation tissue.

persistent cranial nerve abnormalities (2). Mortality due to invasive mycosis of the lateral skull base was reported in literature to be approximately $27 \%$ (6). Therefore, a multi-disciplinary team approach is needed in the treatment of SBO. The main treatment aim should be culture directed therapy (3) and long term antibiotics (8). In summary, SBO should be suspected in an elderly patient who is a diabetic presenting with otalgia and otorrhoea and if granulation tissue is seen in the ear canal (8). A high index of suspicion is required for diagnosing a fungal cause of lateral SBO especially if there is an intractable course of treatment.

\section{REFERENCES}

1. Volsky PG, Hillman TA. Fungal skull base osteomyelitis: Emerging microbial identification techniques. Laryngoscope 2017; 127(1): E5-E7.

2. Blyth C, Gomes L, Sorrell T, Da Cruz M, Sud A, Chen SA. Skull-base osteomyelitis: fungal vs. bacterial infection. Clinical Microbiology and Infection 2011; 17(2): 306-11.

3. Mortazavi MM, Khan MA, Quadri SA, et al. Cranial Osteomyelitis: A Comprehensive Review of Modern Therapies. World Neurosurgery 2018; 111: 142-53.

4. Meltzer PE, Kelemen G. Pyocyaneous osteomyelitis of the temporal bone, mandible and zygoma. Laryngoscope 1959; 69(10): 1300-16.

5. Gleeson MJ, Clarke RC. Scott-Brown's Otorhinolaryngology: Head and Neck Surgery. 7th Ed. CRC Press 2008.

6. Stodulski D, Kowalska B, Stankiewicz C. Otogenic skull base osteomyelitis caused by invasive fungal infection. European Archives of Oto-Rhino-Laryngology and Head \& Neck. 2006; 263(12): 1070-6.

7. Yao M, Messner AH. Fungal malignant otitis externa due to Scedosporium apiospermum. Annals of Otology, Rhinology \& Laryngology $2001 ; 110(4): 377-80$.

8. Carfrae MJ, Kesser BW. Malignant otitis externa. Otolaryngologic Clinics of North America 2008; 41(3): 537-49.

9. Bowles PF, Perkins V, Schechter E. Fungal malignant otitis externa. BMJ Case Reports 2017; 2017: bcr2016218420.

10. Shelton JC, Antonelli PJ, Hackett R. Skull base fungal osteomyelitis in an immunocompetent host. Otolaryngology - Head and Neck Surgery $2002 ; 126(1): 76-8$. 